\title{
INTERPOLATION AND INTEGRATION BASED ON AVERAGED VALUES
}

\author{
BORISLAV BOJANOV \\ Department of Mathematics, University of Sofia \\ Blvd. James Bourchier 5, 1164 Sofia, Bulgaria \\ E-mail:boris@fmi.uni-sofia.bg
}

Dedicated to the 70th anniversary of Professor Zbigniew Ciesielski

\begin{abstract}
We discuss recent results on constructing approximating schemes based on averaged values of the approximated function $f$ over linear segments. In particular, we describe interpolation and integration formulae of high algebraic degree of precision that use weighted integrals of $f$ over non-overlapping subintervals of the real line. The quadrature formula of this type of highest algebraic degree of precision is characterized.
\end{abstract}

1. Introduction. We are going to consider some classical questions in Approximation theory from a certain non-standard point of view. Namely, we shall formulate and discuss fundamental problems in interpolation and approximate integration based on a finite number of averaged values instead of the standard data of point evaluations.

According to the Weierstrass theorem, every continuous function can be approximated by an algebraic polynomial with any preassigned accuracy. In addition, it was universally accepted in the precomputer time that the algebraic polynomials are nice functions, easy to evaluate, to differentiate and integrate. That is why the approach that was mostly appreciated in the classical numerical analysis was the one based on the idea of highest algebraic degree of precision. In other words, a numerical method from a given type was considered good and reasonable if it could reproduce polynomials of the highest possible degree. We shall follow this approach here although it is not so strongly dominating in the contemporary numerical analysis and approximation theory. However, it still generates nice theoretical problems requiring sometime deep and hard mathematics and admitting extremely beautiful treatment.

2000 Mathematics Subject Classification: 41A05, 65D32.

The author was supported by the Ministry of Education and Science of Bulgaria under Project No. MM 1402/2004.

The paper is in final form and no version of it will be published elsewhere. 
We shall use the common notation $\pi_{n}$ for the set of all univariate algebraic polynomials of degree less than or equal to $n$. When studying multivariate problems we shall use the notation $\Pi_{n}\left(\mathbb{R}^{d}\right)$ for the set of all algebraic polynomials of total degree $n$ in $d$ variables. For example, the space of polynomials of total degree $n$ in two variables $x, y$ is

$$
\pi_{n}\left(\mathbb{R}^{2}\right):=\left\{\sum_{0 \leq i+j \leq n} c_{i j} x^{i} y^{j}: c_{i j} \text { are real }\right\} .
$$

Some of the results we are going to present can be extended to cover approximation by Tchebycheff systems. Recall that the functions $u_{1}, \ldots, u_{n}$ form a Tchebycheff system on $[a, b]$ if every non-zero generalized polynomial

$$
c_{1} u_{1}(x)+\cdots+c_{n} u_{n}(x)
$$

with respect to $\left\{u_{k}\right\}$ has at most $n-1$ distinct zeros in $[a, b]$.

This article concerns the classical Gauss quadrature formula and its extensions. In 1814 Carl Friedrich Gauss proved that for every given finite interval $[a, b]$ and every $n$ there exists only one quadrature formula of the form

$$
\int_{a}^{b} f(x) d x \approx \sum_{k=1}^{n} A_{k} f\left(x_{k}\right)
$$

which integrates exactly all algebraic polynomials $f$ of degree less than or equal to $2 n-1$. Clearly $2 n-1$ is the highest possible degree that can be achieved by a formula with $n$ nodes. This remarkable result has been extended in many directions. A wonderful extension was given by M. Krein in 1951 (see [16]). He proved the following.

Theorem A (Krein's Theorem). Let $u_{1}, \ldots, u_{2 n}$ be any Tchebycheff system of continuous functions on $[a, b]$. Then there exists a unique set of nodes $x_{1}<\cdots<x_{n}$ and coefficients $\left\{c_{k}\right\}$ such that

$$
\int_{a}^{b} u(x) d x \approx \sum_{k=1}^{n} c_{k} u\left(x_{k}\right)
$$

for every $u \in \operatorname{span}\left\{u_{1}, \ldots, u_{2 n}\right\}$.

In both cases (Gauss, Krein) a standard type of information is used, namely, a set of function values $f\left(x_{1}\right), \ldots, f\left(x_{n}\right)$. In practice, usually these values are obtained as a result of certain experiment or measurement, or just computed. So, there is enough evidence to suppose that the values are not exact, that they are given with a certain error. Then one may consider $f\left(x_{k}\right)$ as an averaged value of the function $f$ on a small subinterval around $x_{k}$. In other words, one may take

$$
f\left(x_{k}\right)=\frac{1}{\delta_{k}+\varepsilon_{k}} \int_{x_{k}-\varepsilon_{k}}^{x_{k}+\delta_{k}} f(x) d x,
$$

with some $\varepsilon_{k}>0, \delta_{k}>0$. This argument could justify the problem of constructing numerical methods that are based directly on averaged values of the studied function on finite number of subintervals. Actually, similar reasoning as in the above has led in sixties to the development of a new field in numerical analysis, called "interval analysis". We shall concentrate here just on one particular problem - the interval counterpart of the famous formula of Gauss. 


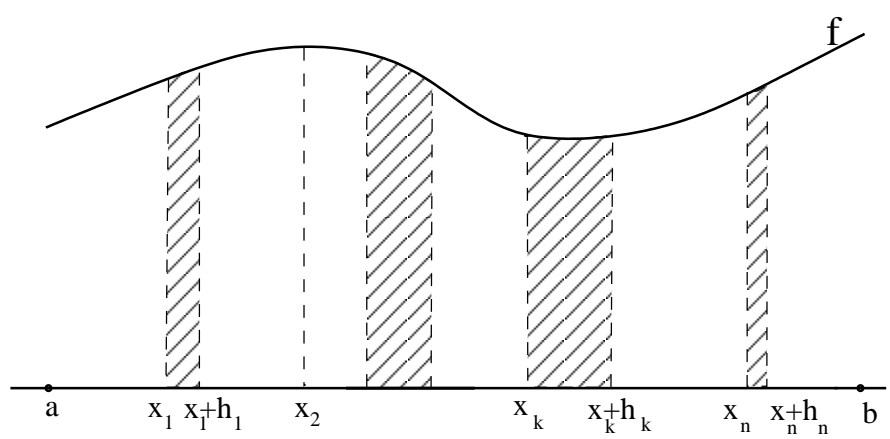

Fig. 1. Interval quadrature

Problem. Given any $h_{1} \geq 0, \ldots, h_{n} \geq 0$ such that

$$
h_{1}+\cdots+h_{n}<b-a
$$

does there exist a formula of the form

$$
\int_{a}^{b} f(x) d x \approx \sum_{k=1}^{n} a_{k} \cdot \frac{1}{h_{k}} \int_{x_{k}}^{x_{k}+h_{k}} f(x) d x
$$

with $a \leq x_{1} \leq x_{1}+h_{1} \leq x_{2} \leq x_{2}+h_{2} \leq \cdots \cdots \leq x_{n} \leq x_{n}+h_{n} \leq b$, which is exact for every $f \in \pi_{2 n-1}$ ?

It is not difficult to see that the highest degree of precision that can be achieved by a formula of type (1) is indeed $2 n-1$. To do this one has to construct a polynomial $p$ of degree $2 n$ such that

$$
\int_{x_{k}}^{x_{k}+h_{k}} p(x) d x=0, \quad k=1, \ldots, n,
$$

and

$$
p(x)>0 \quad \text { on }[a, b] \backslash \cup_{k=1}^{n}\left[x_{k}, x_{k}+h_{k}\right] .
$$

Clearly, the conditions on $p$ imply $\int_{a}^{b} p(x) d x>0$ while the approximating sum in (1) is zero. The existence of such a polynomial follows from an interpolation theorem we are going to prove in the sequel (see Proposition 2 and the remark there).

Note that in the particular case when all $h_{k}$ are equal to zero formula (1) reduces to the Gauss formula since

$$
\lim \frac{1}{h_{k}} \int_{x_{k}}^{x_{k}+h_{k}} f(x) d x=f\left(x_{k}\right) \quad \text { as } \quad h_{k} \rightarrow 0 .
$$

Studying extensions of classical integration and interpolation problems based on averaged values is of independent interest. But one can find serious motivation to justify such research in practice and also in the extremely elegant solutions of problems of this kind, in particular in the multivariate case. Before starting the consideration of our main problem (1) we find it worthwhile to recall first several approximation results which deal with data consisting of line integrals. They illustrate the elegance and the naturality of the interval setting (especially in the bivariate case) and thus could motivate other mathematicians to continue the research in this interesting field. 
2. Motivation for studying interval formulae. Formula (1) is a natural extension of a fundamental classical quadrature formula and this is itself a motivation to study the question of complete characterization of the optimal subintervals. In the multivariate case, one can supply much more reasons and motivation to study numerical methods based on averaged values. For example, integrals over linear segments appear as output in tomography, electronic microscopy, geophysics, and hence, a direct usage of the output data for reconstruction of the function into consideration, or functionals of it, is a quite natural task. Problems based on averaged values have been considered before and in many cases the interval setting has led to remarkable results. We shall mention first the wonderful interpolation theorem proved by Hakop Hakopian. For the sake of simplicity we shall formulate it in the bivariate case. Its general multivariate setting can be found in [15] (see also [2], Chapter 12).

2.1. Interpolation on the plane. We shall consider integrable functions $f(x, y)$ on a convex domain $\Omega$ in $\mathbb{R}^{2}$. For the sake of simplicity, we suppose that $0 \in \Omega$ and that $f(x, y)$ vanishes outside $\Omega$. Any pair of parameters $(t, \theta)$ defines a line

$$
I(t, \theta):=\{(x, y): x \cos \theta+y \sin \theta=t\} .
$$

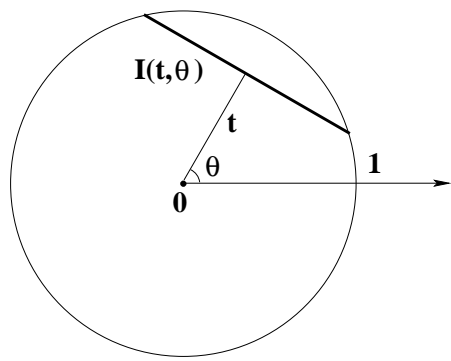

Fig. 2. Radon projection

We assume that $\theta \in[0, \pi)$ and

$$
I(t, \theta) \cap \Omega \not \equiv \emptyset .
$$

The projection $\int_{I} f$ of $f$ along the line $I(t, \theta)$ is defined by

$$
\int_{I} f:=\int_{-\infty}^{+\infty} f(t \cos \theta-s \sin \theta, t \sin \theta+s \cos \theta) d s .
$$

In the case $\Omega$ is the unit disk, $P_{f}(t, \theta)$ is called Radon projection.

Hakopian's Interpolation TheOrem. Given a convex set $\Omega$, any $n+2$ distinct points $\mathbf{x}_{0}, \ldots, \mathbf{x}_{n+1}$ on the boundary $\partial \Omega$ of $\Omega$, and numbers $\left\{\gamma_{k}\right\}_{0}^{n+1}$, there exists a unique algebraic polynomial $P \in \Pi_{n}\left(\mathbb{R}^{2}\right)$ such that

$$
\int_{I_{k}} P=\gamma_{k}, \quad k=1, \ldots, N
$$

where $N$ is the dimension of $\Pi_{n}\left(\mathbb{R}^{2}\right)$, i.e., $N=(n+1)(n+2) / 2$, and $I_{1}, \ldots, I_{N}$ are the linear segments that join any two distinct points from the set $\mathbf{x}_{0}, \ldots, \mathbf{x}_{n+1}$. 


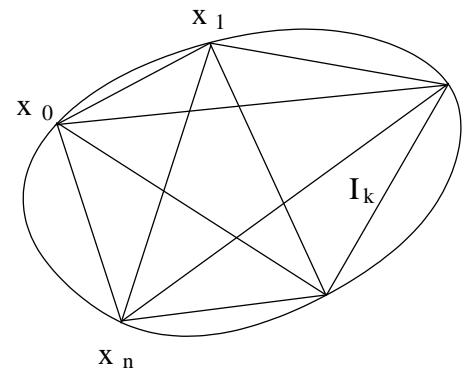

Fig. 3. Hakopian's interpolation

The bivariate case of Hakopian's theorem follows also from an interpolation result given by Cavaretta, Micchelli, Sharma [12] and the particular case of equally spaced points $\mathbf{x}_{0}, \ldots, \mathbf{x}_{n+1}$ on the unit disk was considered earlier by Marr [18].

The following elegant proof of the solvability of the bivariate interpolation problem (2) is worth mentioning. It was communicated to us by Hakopian. Let us first recall an important definition.

Given the real function $\rho(t)$ on $\mathbb{R}$ and a parameter $\theta \in[0, \pi)$ we define on $\mathbb{R}^{2}$ the associated ridge function $\rho(\theta ; x, y)$ with direction $\theta$ in the following way

$$
\rho(\theta ; x, y):=\rho(x \cos \theta+y \sin \theta) .
$$

In other words, $\rho(\theta ; x, y)=\rho((\mathbf{x} \cdot \xi))$ where, as usual, $(\mathbf{x} \cdot \xi)$ denotes the scalar (dot) product of the vectors $\mathbf{x}=(x, y)$ and $\xi=(\cos \theta, \sin \theta)$. Clearly the ridge function is constant along any line of direction $\theta+\pi / 2$.

To show the existence and the uniqueness of the solution of the corresponding linear system (2), we shall construct a Lagrangean basis of ridge polynomials for $\Pi_{n}\left(\mathbb{R}^{2}\right)$, i.e., we shall show that for every fixed $k, 1 \leq k \leq N$, there exists a ridge polynomial $Q_{k}$ such that

$$
\int_{I_{i}} Q_{k}=\delta_{i k}, \quad i=1, \ldots, N,
$$

where $\delta_{i k}$ is the Kronecker symbol. Clearly the polynomials $\left\{Q_{k}\right\}_{k=1}^{N}$ are linearly independent. Since the number of the polynomials $\left\{Q_{k}\right\}_{k=1}^{N}$ equals the dimension of $\Pi_{n}\left(\mathbb{R}^{2}\right)$, we conclude that $\left\{Q_{k}\right\}_{k=1}^{N}$ constitute a basis in $\Pi_{n}\left(\mathbb{R}^{2}\right)$.

It remains to construct the polynomials $Q_{k}$. In order to do this, we fix $k$ and choose the coordinate system $O x y$ so that the segment $I_{k}$ lies on the $y$ axis (see Fig. 4). Without loss of generality we may assume that $\mathbf{x}_{n}$ and $\mathbf{x}_{n+1}$ are the end points of the linear segment $I_{k}$. Clearly, the orthogonal projections of the points $\mathbf{x}_{n}$ and $\mathbf{x}_{n+1}$ on the real axis coincide with the origin. Let us denote by $t_{0}, \ldots, t_{n-1}$ the orthogonal projections of the remaining points $\mathbf{x}_{0}, \ldots, \mathbf{x}_{n-1}$, respectively, and set $t_{n}=0$. Let $Q(x, y)=q(x)$ be a ridge polynomial with direction $\theta=0$, i.e., which takes constant values on any line parallel to the $y$ axis. Take the linear segment $I:=\left[\mathbf{x}_{i}, \mathbf{x}_{j}\right]$ connecting the points $\mathbf{x}_{i}$ and $\mathbf{x}_{j}$. Now, observe that the integral of $Q$ over $I$ will be zero provided the univariate integral $\int_{t_{i}}^{t_{j}} q(t) d t$ equals zero. Thus, in order to construct a ridge polynomial $Q_{k}(x, y)=q_{k}(x)$ which satisfies the interpolation conditions (3), it suffices to find a univariate polynomial 


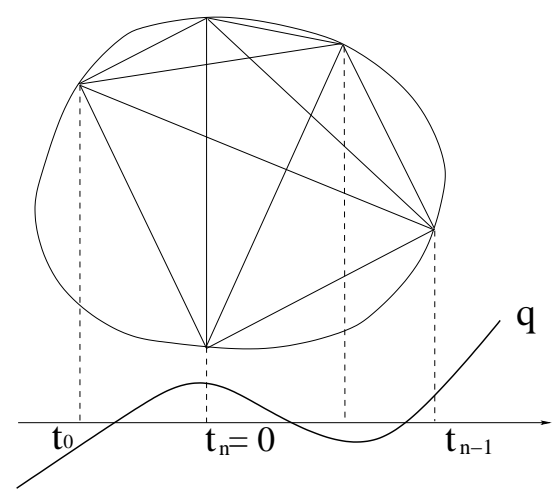

Fig. 4. Lagrangean ridge polynomial

$q_{k}(x)$ of degree $n$ such that $q_{k}(0)=1 /\left|I_{k}\right|$ and

$$
\int_{t_{i-1}}^{t_{i}} q_{k}(t) d t=0, \quad i=1, \ldots, n .
$$

But the latter is a simple univariate interpolation problem which has a unique solution $q_{k}$. Then the ridge polynomial $Q(x, y):=q_{k}(x)$ will satisfy the required interpolation conditions. Evidently the equation of this polynomial can be now rewritten with respect to any preassigned coordinate system (one and the same for each $k$ ), and let us call it $Q_{k}$. Therefore, we can construct a Lagrangean basis $\left\{Q_{k}\right\}_{k=1}^{N}$ for the polynomial space $\Pi_{n}\left(\mathbb{R}^{2}\right)$. Then the unique solution $P$ of the interpolation problem (2) is given by

$$
P(x, y)=\sum_{k=1}^{N} \gamma_{k} Q_{k}(x, y) .
$$

REMARK. It follows from the above that any polynomial $P$ from $\Pi_{n}\left(\mathbb{R}^{2}\right)$ can be represented as a linear combination of $N=(n+1)(n+2) / 2$ ridge polynomials. Another interesting result along this line is the following proposition, established first by Vostretsov and Kreines [30].

For any given set of $n+1$ non-co-linear unit vectors $\xi_{j}$ and a polynomial $P \in \Pi_{n}\left(\mathbb{R}^{2}\right)$, there exist univariate polynomials $r_{0}, \ldots, r_{n}$ of degree $n$ such that

$$
P(\mathbf{x})=\sum_{j=0}^{n} r_{j}\left(\mathbf{x} \cdot \xi_{j}\right) .
$$

It was shown recently that for any fixed polynomial $P$ of degree $n$ one can find appropriate directions $\theta_{1}, \ldots, \theta_{n}$ such that $P$ can be represented as a linear combination of $n$ ridge polynomials with directions $\theta_{1}, \ldots, \theta_{n}$, respectively. A simpler proof and an improvement concerning the choice of the directions $\left\{\theta_{k}\right\}$ was given in [9]. Oskolkov noticed in [25] that the number $n$ cannot be further diminished.

As is well known, the standard pointwise interpolation problem in the multivariate case is not always poised, that is, the matrix of the corresponding system of linear equations is not always invertable. There are only a few configurations of nodes that produce 
uniquely solvable interpolation problem. Almost all of them, like the classical Radon scheme and its particular case of Chang-Yao configuration, are based on a standard application of the Bezout theorem. Recently, new configurations were found in [10], using another approach, liberated from the limitation of the Bezout theorem.

Hakopian's interpolation is based on data of linear integrals. Such data is a natural output in tomography and electronic microscopy. In this light, Hakopian's theorem is not only a wonderful theoretical result, it solves also a problem of practical importance: to recover a function from its Radon projections (i.e., from a finite number of line integrals along chords in the disk). This is a serious reason to study other approximation schemes based on line integrals. Recently, it was shown in [9] how to construct a regular interpolation scheme based on $N$ linear integrals taken in $n+1$ distinct directions on parallel chords: one chord in one direction, 2 parallel chords in another direction, 3 parallel chords in a third direction and so on, $n+1$ parallel chords in a direction different from the previous ones (see Fig. 5). The number of chords is exactly $N$, i.e., it equals the dimension of the set of polynomials of degree $n$. The configuration of chords can be described by a set of given angles $\theta_{0}<\cdots<\theta_{n}$ in $[0, \pi)$ and a triangular matrix $T=\left\{t_{k i}\right\}$ of points

$$
t_{k k}<\cdots<t_{k n}, \quad k=0, \ldots, n,
$$

associated with the angles. The problem is to characterize all locations of the nodes $\left\{t_{k j}\right\}$ for which the interpolation of the data $\left\{\int_{I\left(\theta_{k} ; t_{k j}\right)} f\right\}$ by polynomials of degree $n$ is poised.

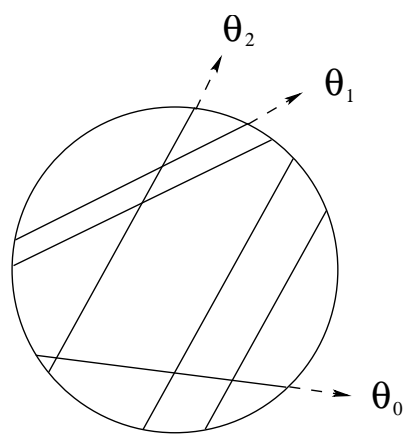

Fig. 5. Regular configuration

The matrices

$$
\mathbf{U}_{k}:=\left(\begin{array}{cccc}
U_{k}\left(t_{k k}\right) & U_{k+1}\left(t_{k k}\right) & \cdots & U_{n}\left(t_{k k}\right) \\
U_{k}\left(t_{k, k+1}\right) & U_{k+1}\left(t_{k, k+1}\right) & \cdots & U_{n}\left(t_{k, k+1}\right) \\
\vdots & \vdots & \cdots & \vdots \\
U_{k}\left(t_{k n}\right) & U_{k+1}\left(t_{k n}\right) & \cdots & U_{n}\left(t_{k n}\right)
\end{array}\right)
$$

play a crucial role in the characterization of the interpolation problem.

THEOREM B. For given angles $0 \leq \theta_{0}<\cdots<\theta_{n}<\pi$ and associated points $T=$ $\left\{t_{k i}\right\}_{k=0, i=k}^{n}$, the interpolation problem

$$
\int_{I\left(\theta_{k}, t_{k i}\right)} P=\gamma_{k i}, \quad k=0, \ldots, n, i=k, \ldots, n, \quad P \in \Pi_{n}\left(\mathbb{R}^{d}\right)
$$


is poised if and only if

$$
\operatorname{det} \mathbf{U}_{k} \neq 0 \quad \text { for } \quad k=1, \ldots, n \text {. }
$$

Note that the conditions for the regularity of the interpolation problem are expressed only through the points $\left\{t_{i j}\right\}$. They are the same for any choice of the directions $\left\{\theta_{k}\right\}$. It would be interesting to find explicitly some particular configuration of points for which the determinants $\operatorname{det} \mathbf{U}_{k}$ are non-zero.

The next proposition can be derived as a particular case of Theorem B.

Theorem C. Let $0 \leq \theta_{0}<\cdots<\theta_{n}<\pi$ be any given angles and let $T$ be a set of arbitrary $n+1$ distinct points in $(-1,1)$. Then there exists a numbering $t_{0}, \ldots, t_{n}$ of the points from $T$ so that the interpolation problem

is poised in $\Pi_{n}\left(\mathbb{R}^{2}\right)$.

$$
\int_{I\left(\theta_{i}, t_{j}\right)} P=\gamma_{i j}, \quad i=0, \ldots, n, j=i, \ldots, n,
$$

Further development of recovery of polynomials from their Radon projections is presented in [11], where the chords are taken in equal number in each direction and where parameters that define poised interpolation are given explicitly.

2.2. Multivariate integration. Almost 200 hundred years after Gauss proved his celebrated formula there is no yet a multivariate analog of this formula even for simplest domains in the plane. We mean, there is no result saying that for a given domain $\Omega$ and for every $n$ a set of nodes $\left\{\left(x_{1}, y_{1}\right), \ldots\left(x_{n}, y_{n}\right)\right\}$ is completely characterized such that the cubature formula

$$
\int_{\Omega} f(\mathbf{x}) d \mathbf{x} \approx \sum_{k=1}^{n} c_{k} f\left(x_{k}, y_{k}\right)
$$

has a highest algebraic degree of precision with respect to the space of algebraic polynomials in two variables. The characterization of the extremal nodes is an extremely difficult problem. Partial characterization is given through the common zeros of orthogonal polynomials on $\Omega$ (see, for example, [22] and the recent book [13]). Explicit formulae of highest degree are derived for the square with the Tchebycheff weight (see [20], [31], [6]). The uniqueness of the set of extremal nodes is not established yet for any domain in the plane.

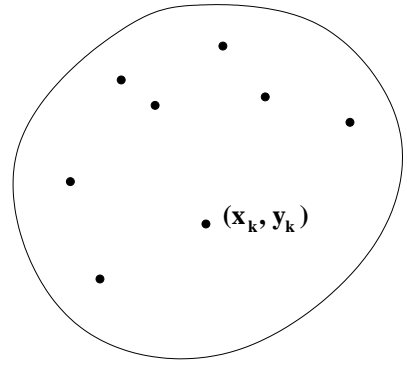

Fig. 6. Classical pointwise setting

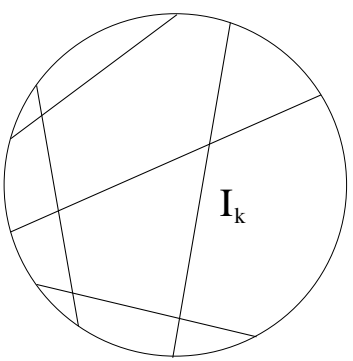

Fig. 7. The new interval setting

Consider now an alternative approach. Let $\mathbf{B}$ denote the unit disk on the plane, i.e., $\mathbf{B}:=\left\{(x, y): x^{2}+y^{2} \leq 1\right\}$. For given set of chords $I_{1}, \ldots, I_{n}$ in $\mathbf{B}$, we shall study 
cubature formulae of the form

$$
\int_{\mathbf{B}} f(\mathbf{x}) d \mathbf{x} \approx \sum_{k=1}^{n} C_{k} \int_{I_{k}} f .
$$

The question is: Given n, what is the highest degree of precision one can get by a formula (4)?

Clearly, the maximal algebraic degree of precision of a formula of type (4) (in brief, $\operatorname{ADP}(4))$ satisfies

$$
\operatorname{ADP}(4) \leq 2 n-1
$$

Indeed, if $\ell_{k}(x, y)=0$ is the equation of the line containing the segment $I_{k}$, then

$$
\omega(x, y):=\prod_{k=1}^{n} \ell_{k}(x, y)
$$

is a polynomial of total degree $n$ and obviously $\omega^{2}(x, y) \in \Pi_{2 n}\left(\mathbb{R}^{2}\right)$, it vanishes on $I_{k}$, $k=1, \ldots, n$, and

$$
\omega^{2}(x, y) \geq 0 \quad \text { on } \mathbf{B}
$$

Therefore

$$
\int_{\mathbf{B}} \omega^{2}(\mathbf{x}) d \mathbf{x}>0 \quad \text { while } \quad \sum_{k=1}^{n} C_{k} \int_{I_{k}} \omega^{2}=0 .
$$

Thus, the highest degree one can hope to get with a formula of form (4) is $2 n-1$. The following was proved in [8].

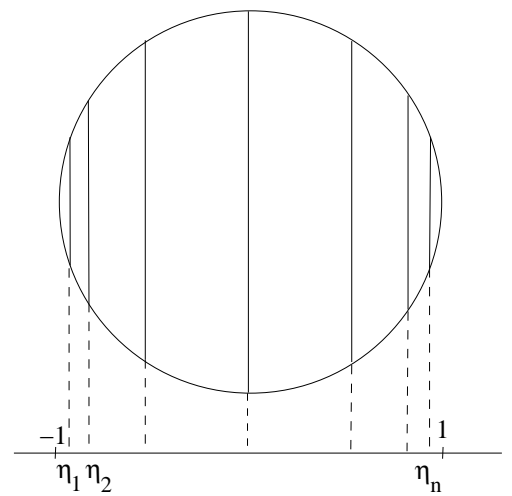

Fig. 8. Gaussian configuration

THEOREM D. For every $n$ there exists a unique (up to rotation) cubature formula of type (4) which is exact for all bivariate polynomials of degree less than or equal to $2 n-1$. The extremal cubature formula is based on chords that are parallel to Oy axis and pass through the zeros $\left\{\eta_{k}\right\}_{k=1}^{n}$ of the Tchebycheff polynomial of the second kind $U_{n}(x)$. The formula is given explicitly by

$$
\iint_{\mathbf{B}} f(x, y) d x d y \approx \sum_{k=1}^{n} A_{k} \int_{-\sqrt{1-\eta_{k}^{2}}}^{\sqrt{1-\eta_{k}^{2}}} f\left(\eta_{k}, y\right) d y
$$


with

$$
A_{k}=\frac{\pi}{n+1} \sin \frac{k \pi}{n+1}, \quad k=1, \ldots, n .
$$

Extension of the last result to the general multivariate case is also given in [8].

3. Interpolation problem. The examples in the previous section show that one can find enough motivation to study approximation problems with respect to data consisting of integral evaluations. We shall consider here interpolation by univariate polynomials based on integrals over subintervals. The following proposition can be easily proved.

Proposition 1 (The Lagrangean case). Given the values $\left\{\gamma_{k}\right\}_{k=0}^{n}$ and $n+1$ non-overlapping intervals

$$
\Delta_{k}:=\left[a_{k}, b_{k}\right], \quad k=0, \ldots, n,
$$

on the real line $\mathbb{R}$, there exists a unique $p \in \pi_{n}$ such that

$$
\int_{\Delta_{k}} p(t) d t=\gamma_{k}, \quad k=0, \ldots, n .
$$

The proof is standard: If a polynomial $p$ from $\pi_{n}$ satisfies the homogeneous conditions (i.e., with $\gamma_{k}=0$, all $k$ ), then it would follow that $p$ vanishes at least at one point from each subinterval $\Delta_{k}, k=0, \ldots, n$, and consequently $p \equiv 0$.

A natural extension of the above interpolation problem would be to impose conditions of the same kind on the derivatives of $p$, that is, to consider a Hermitean type of interpolation. The following very particular version was proved in [3].

Proposition 2. Let $\nu_{1}, \ldots, \nu_{m}$ be any system of integers such that $\nu_{k} \in\{1,2\}$. Let $n:=\nu_{1}+\cdots+\nu_{m}-1$. Then for every given set of $n$ non-overlapping intervals

$$
\left[a_{k}, b_{k}\right], \quad k=0, \ldots, n,
$$

and values $f_{k 0}, f_{k 1}, k=1, \ldots, m$, there exists a unique polynomial $p \in \pi_{n}$, satisfying the interpolation conditions

$$
\frac{1}{b_{k}-a_{k}} \int_{a_{k}}^{b_{k}} p^{(j)}(t) d t=f_{k j}, \quad k=1, \ldots, m, j=0, \nu_{k}-1 .
$$

Proof. As in the previous problem we need to show that the corresponding homogeneous interpolation problem (with $f_{k j}=0$ ) admits only the zero solution $p \equiv 0$. Indeed, let us assume that a polynomial $p$ from $\pi_{n}$ satisfies

$$
\frac{1}{b_{k}-a_{k}} \int_{a_{k}}^{b_{k}} p^{(j)}(t) d t=0, \quad k=1, \ldots, m, j=0, \nu_{k}-1,
$$

If $a_{k}=b_{k}$, then the corresponding interpolation conditions reduce to $p\left(a_{k}\right)=0$ (and $\left.p^{\prime}\left(a_{k}\right)=0\right)$, in case $\left.\nu_{k}=2\right)$. Thus, they imply existence of at least $\nu_{k}$ zeros of $p$ in $\left[a_{k}, b_{k}\right]$. Assume that $a_{k}<b_{k}$. Then the condition $\int_{a_{k}}^{b_{k}} p(t) d t=0$ implies that $p$ should change its sign in $\left(a_{k}, b_{k}\right)$ and thus $p$ has a zero there. If, in addition, $\int_{a_{k}}^{b_{k}} p^{\prime}(t) d t=0$, then $p\left(a_{k}\right)=p\left(b_{k}\right)$ and evidently $p$ should have an even number of zeros in $\left(a_{k}, b_{k}\right)$ (except in the trivial case $p\left(a_{k}\right)=p\left(b_{k}\right)=0$ when $p$ vanishes at the end points of $\left[a_{k}, b_{k}\right]$ and 
changes sigh inside the interval). Therefore, $p$ has at least one zero in $\left[a_{k}, b_{k}\right]$ if $\nu_{k}=1$, or at least two zeros if $\nu_{k}=2$. Hence, $p$ will have at least $n+1$ zeros, counting multiplicities, and this implies $p \equiv 0$ what was to be shown.

REMARK. Taking $\nu_{1}=\cdots=\nu_{n}=2$ in the last proposition we derive the existence of a polynomial $p \in \pi_{2 n}$ such that

$$
\int_{a_{k}}^{b_{k}} p(x) d x=\int_{a_{k}}^{b_{k}} p^{\prime}(x) d x=0, \quad k=1, \ldots, n .
$$

Since $p$ has 2 zeros in each subinterval $\left[a_{k}, b_{k}\right]$, we conclude that $p(x)>0$ outside $\cup_{1}^{n}\left[a_{k}, b_{k}\right]$. The existence of such a polynomial was needed to show that the algebraic degree of precision of (1) is less than $2 n$.

The general case, of arbitrary multiplicities $\left\{\nu_{k}\right\}$, was studied in [17]. It is stated there that the Hermite interpolation problem with averaged value conditions (i.e., Proposition 2 with arbitrary multiplicities $\left.\left\{\nu_{k}\right\}\right)$ is solvable. Unfortunately the proof given there is not correct. Even more, the following counterexample communicated to us by Petar Petrov shows that such a proposition cannot be true.

Counterexample. Consider the polynomial

$$
f(x)=\frac{x^{4}}{4}-\frac{x^{2}}{2}+\frac{7}{60} .
$$

We compute

$$
\int_{-1}^{1} f(t) d t=\int_{-1}^{1} f^{\prime}(t) d t=\int_{-1}^{1} f^{\prime \prime}(t) d t=\int_{-1}^{1} f^{\prime \prime \prime}(t) d t=0 .
$$

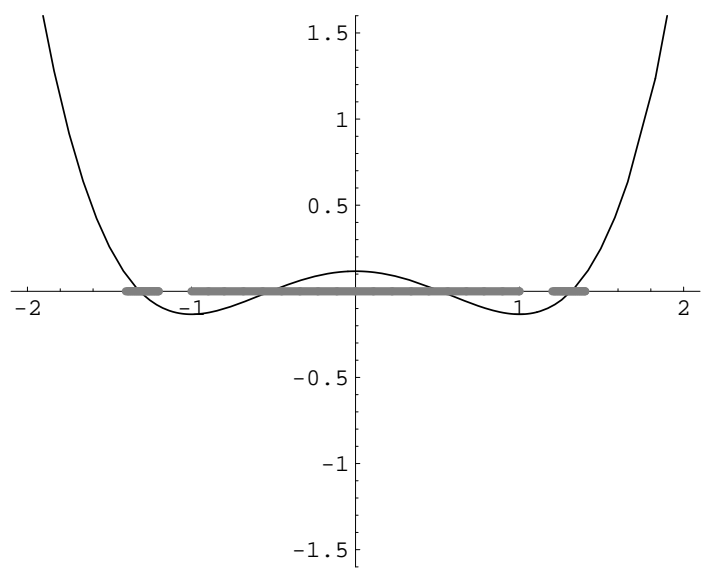

Fig. 9. Counterexample

Further, observe that

$$
f(0)>0, \quad f(-1)=f(1)<0 .
$$

Thus, $f$ changes sign at some points $x_{1}<-1$ and $x_{2}>1$ (see Fig. 9). Then one can 
choose small subintervals $\left[a_{1}, b_{1}\right],\left[a_{2}, b_{2}\right]$ around $x_{1}$ and $x_{2}$, respectively, so that

$$
\int_{a_{k}}^{b_{k}} f(t) d t=0, \quad k=1,2 .
$$

Adding also the conditions

$$
\int_{-1}^{1} f^{(j)}(t) d t=0 \quad \text { for } \quad j=0,1,2,3,
$$

we see that the non-zero polynomial $f$ of degree 4 satisfies 6 averaged value interpolation conditions of Hermitean type on 3 subintervals. Thus the interpolation matrix is singular.

The condition in Proposition 1 that the subintervals $\left\{\left[a_{k}, b_{k}\right]\right\}$ are non-overlapping is essential. A simple example can be constructed that shows that the interpolation cannot be regular if we allow the subintervals to overlap. Indeed, take the non-zero polynomial $p(t)=t$. It satisfies the zero mean value conditions $\int_{-k}^{k} p(t) d t=0$ on the overlapping subintervals $[-k, k], k=1,2, \ldots, n$, for any $n$, as large as we want.

One can slightly extend Proposition 1, allowing restricted overlapping. The following interpolation result was proved in [3].

Assume that $[a, b]$ is a fixed finite interval and $\mu(t)$ is a given weight on $[a, b]$ (i.e., a Lebesgue integrable non-negative function such that $\int_{\alpha}^{\beta} \mu(t) d t>0$ for each subinterval $[\alpha, \beta] \subset[a, b]$ of positive length). For subintervals $I, J$ of $[a, b]$ we shall write $I<J$ to denote the fact that $I \cap J$ has no interior points and $x \leq y$ for each $x \in I$ and $y \in J$. The length of $I$ will be denoted by $|I|$.

Proposition 3. Let $u_{1}, \ldots, u_{2 n}$ be a given Tchebycheff system of continuous functions on $[a, b]$ and $I_{1}<\ldots<I_{n}, J_{1}<\ldots<J_{n}$ be given subintervals of $[a, b]$ such that $\left|I_{i}\right|=\left|J_{i}\right|=h, i=1, \ldots, n$, and $I_{i} \not \equiv J_{j} \forall i, j=1, \ldots, n$. Then the homogeneous interpolation problem

$$
\int_{I_{i}} \mu(t) u(t) d t=\int_{J_{i}} \mu(t) u(t) d t=0, i=1, \ldots, n,
$$

has only the zero solution $u \equiv 0$ in span $\left\{u_{1}, \ldots, u_{2 n}\right\}$.

The proof can be seen in [3] (Lemma 2).

We already mentioned that the Hermitean interpolation problem in its averaged value setting is not regular, in general. But one can formulate multiple interpolation conditions of different kind, under which the problem is solvable in quite general form. We recall here a result of this type from [3] (see Lemma 1). It concerns interpolation with respect to arbitrary Tchebycheff system. We need to recall some definitions.

Let the set of continuous functions $U_{N}=\left\{u_{1}, \ldots, u_{N}\right\}$ constitute a Tchebycheff system on $[a, b]$.

Assume further that $V_{N}=\left\{v_{1}, \ldots, v_{N}\right\}$ (with $v_{1} \equiv 1$ ) is a given Markov system of continuous functions on $[a, b]$. Recall that $V_{N}$ is said to be a Markov system if the functions $\left\{v_{1}, \ldots, v_{k}\right\}$ form a Tchebycheff system for each $k=1, \ldots, N$.

For a given set of ordered non-overlapping subintervals $\left\{\left[a_{k}, b_{k}\right]\right\}$ such that

$$
b_{0}:=a \leq a_{1}<b_{1} \leq \ldots \leq a_{n}<b_{n} \leq b=: a_{n+1},
$$


with $h_{k}:=b_{k}-a_{k}$, we consider the moments $\mu_{k, \lambda}$ of $f$ with respect to $V_{N}$. More precisely,

$$
\mu_{k, \lambda}(f):=\frac{1}{h_{k}} \int_{a_{k}}^{b_{k}} \mu(t) f(t) v_{\lambda}(t) d t .
$$

THEOREM 1. Given the set $\left\{\left[a_{k}, b_{k}\right]\right\}_{1}^{n}$ of non-overlapping subintervals and real numbers $M_{k \lambda}, k=1, \ldots, n, \lambda=0, \ldots, \nu_{k}-1, \sum_{k=1}^{n} \nu_{k}=N$, there exists a unique generalized polynomial $u \in \operatorname{span} U_{N}$ such that

$$
\mu_{k \lambda}(u)=M_{k \lambda}, \quad k=1, \ldots, n, \lambda=0, \ldots, \nu_{k}-1 .
$$

The construction of the interpolating polynomial even in the simplest Lagrangean case (i.e., that in Proposition 1) does not admit an elegant treatment like in the pointwise interpolation. Of course, one can define generalized divided difference with respect to the averaged values

$$
\left\{\mu_{k}:=\frac{1}{b_{k}-a_{k}} \int_{a_{k}}^{b_{k}} f(t) d t\right\}_{k=0}^{n}
$$

as the leading coefficient in the corresponding interpolating polynomial and then write the interpolating polynomial in Newton's form. But there is no simple way of computation of these divided differences. The fundamental Lagrangean polynomials $\left\{\ell_{k}(t)\right\}$, defined by

$$
\int_{a_{j}}^{b_{j}} \ell_{k}(t) d t=\delta_{k j}
$$

can be computed via the recurrence relation (see [17])

$$
\begin{aligned}
& p_{k}(t)=x^{n}-\sum_{j=0, j \neq k}^{n} \ell_{j}(k ; t) \tilde{\mu}_{j}\left[x^{n}\right], \\
& \ell_{k}(t)=\frac{p_{k}(t)}{\mu_{k}\left[p_{k}\right]}
\end{aligned}
$$

where $\ell_{j}(k ; t)$ is the $j$ th fundamental polynomial of degree $n-1$ for the subset of subintervals obtained from the original one, deleting the $k$ th subinterval, and $\left\{\tilde{\mu}_{j}\right\}$ are the averaged values defined for the reduced set of subintervals. This way of construction of the interpolating polynomial also needs serious computation work.

\section{Interval quadrature formulae}

4.1. Existence. Now let us go back to the Gaussian quadrature formula (1) based on averaged values. Having the type fixed, the first question to ask is about the existence of a quadrature of highest algebraic degree of precision (ADP). More precisely, does there exist a quadrature formula using integrals over $n$ subintervals of $[a, b]$ of ADP equal to $2 n-1$. The question was studied in [23], [26], [27], [17]. The following existence theorem was proved in [26]:

Let $\tau_{1}, \ldots, \tau_{n}$ be the nodes of the classical Gauss quadrature formula. Then for every $a_{k}<\tau_{k}$, sufficiently close to $\tau_{k}, k=1, \ldots, n$, there exists $b_{k}, \tau_{k}<b_{k}$, such that the interpolatory quadrature formula based on the subintervals $\left\{\left[a_{k}, b_{k}\right]\right\}_{k=1}^{n}$ is of degree of precision $2 n-1$. 
In other words, in a neighborhood of the Gauss nodes (the zeros of the corresponding orthogonal polynomial) one can find small subintervals that produce interval Gaussian quadrature.

Existence of interval Gaussian quadratures can be derived from a result in [1] which deals with the relation between the Hobby-Rice theorem and the Gauss formula. Let us recall the Hobby-Rice theorem:

Given any system of integrable functions $\varphi_{1}, \ldots, \varphi_{n}$ on $[a, b]$, there exists points $x_{1}<$ $\cdots<x_{m}$ in $(a, b), m \leq n$, such that

$$
\int_{a}^{b} \varphi_{k}(t) \operatorname{sign} \prod_{j=1}^{m}\left(t-x_{j}\right) d t=0 \quad \text { for } \quad k=1, \ldots, n .
$$

In [1], a non-symmetric extension of Hobby-Rice theorem was presented for Tchebycheff systems. To be more clear, and since we shall need it, we give it here in detail.

For given $\mathbf{x}:=\left(x_{1}, \ldots, x_{n}\right), a=: x_{0}<x_{1}<\cdots<x_{n}<x_{n+1}:=b$, and functions $\mu_{i}(t) \geq 0, i=1,2$, set

$$
[f(t)]_{\left(\mu_{1}, \mu_{2}\right)}:= \begin{cases}\mu_{1}(t) f(t) & \text { if } f(t) \geq 0, \\ \mu_{2}(t) f(t) & \text { if } f(t)<0 .\end{cases}
$$

In case $\mu_{1}=\mu_{2} \equiv 1$ we evidently have $[f(t)]_{\left(\mu_{1}, \mu_{2}\right)}=f(t)$. The function

$$
\sigma\left(\mu_{1}, \mu_{2} ; t\right):=\left[\operatorname{sign} \prod_{j=1}^{m}\left(x-x_{j}\right)\right]_{\left(\mu_{1}, \mu_{2}\right)}
$$

is a kind of generalized sign function.

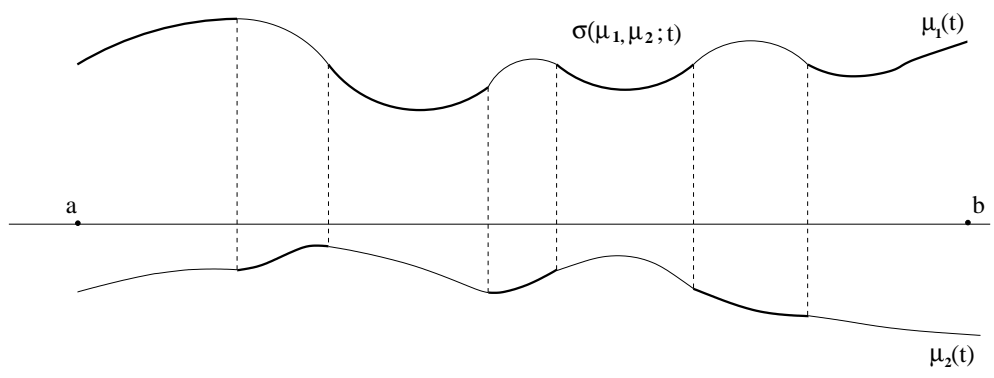

Fig. 10. A generalized sign function

THEOREM 2. Let $\left\{u_{1}, \ldots, u_{n}\right\}$ be an arbitrary Tchebycheff system of integrable functions on $[a, b]$. Then for any fixed pair of weight functions $\mu_{1}(t), \mu_{2}(t)$ on $[a, b]$ there exists a unique system of canonical points $x_{1}<\cdots<x_{m}$ in $(a, b)$ with $m \leq n$ such that

$$
\int_{a}^{b} u_{k}(t)\left[\operatorname{sign} \prod_{j=1}^{m}\left(x-x_{j}\right)\right]_{\left(\mu_{1}, \mu_{2}\right)} d t=0
$$

for each $k=1, \ldots, m$. Moreover, $m=n$.

We shall derive from this theorem existence of interval Gaussian formulae. 
COROLlary 1. Let $u_{1}, \ldots, u_{2 n}$ be any Tchebycheff system on $[a, b]$ of continuous functions. Then there exists $h_{1}>0, \ldots, h_{n}>0$ and a quadrature of type (1) which integrates exactly every generalized polynomial $u(t)$ from $\mathcal{U}_{2 n}:=\operatorname{span}\left\{u_{1}, \ldots, u_{2 n}\right\}$.

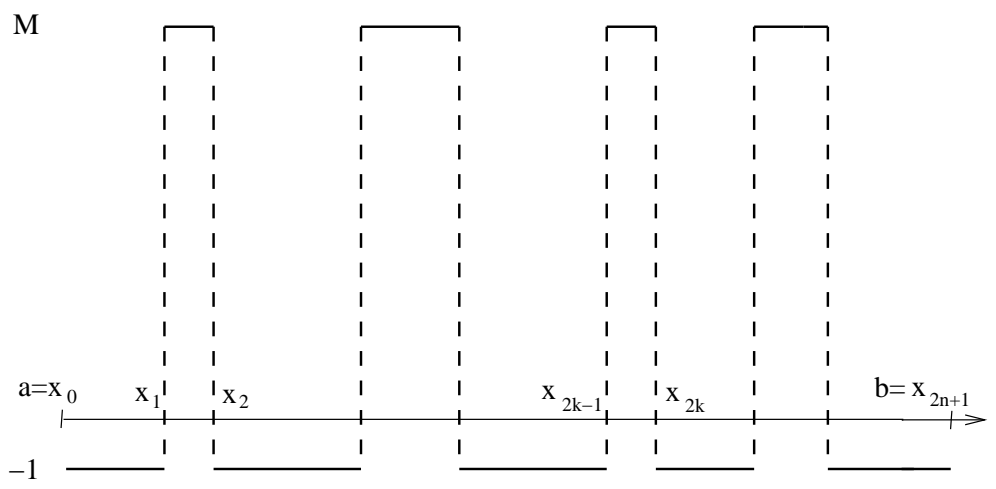

Fig. 11. Non-symmetric sign function

Proof. Take $\mu_{1}(t)=M$ and $\mu_{2}(t)=1$. According to the theorem, there exist points $x_{1}<\cdots<x_{2 n}$ such that

$$
M \sum_{k=1}^{n} \int_{x_{2 k-1}}^{x_{2 k}} u(t) d t=\sum_{j=0}^{n} \int_{x_{2 j}}^{x_{2 j+1}} u(t) d t, \quad \forall u \in \mathcal{U}_{2 n} .
$$

Adding the sum appearing on the left hand side once more to both sides of the equality we obtain

$$
\sum_{k=1}^{n}(M+1) \int_{x_{2 k-1}}^{x_{2 k}} u(t) d t=\int_{a}^{b} u(t) d t, \quad \forall u \in \mathcal{U}_{2 n},
$$

which is an interval Gaussian formula based on the subintervals

$$
\left[x_{2 k-1}, x_{2 k}\right], \quad k=1, \ldots, n .
$$

The existence is proved.

Note here that letting $M$ tend to infinity, one can show that $x_{2 k-1}$ and $x_{2 k}$ tend to the same point $t_{k}$, while $(M+1)\left(x_{2 k}-x_{2 k-1}\right)$ approach some finite numbers $c_{k}$. Thus, as a limit case of the last quadrature we can obtain the Krein extension of the Gauss formula (i.e., Gaussian formula for a Tchebycheff system)

$$
\sum_{k=1}^{n} c_{k} u\left(t_{k}\right)=\int_{a}^{b} u(t) d t, \quad \forall u \in \mathcal{U}
$$

In the existence results we mentioned above the lengths of the subintervals are not specified. These results do not imply the existence of a Gaussian quadrature for any specified set of admissible lengths $\mathbf{h} \in H$. An existence theorem of a quite general form was proved in [3]. We shall give it below. First we pay attention to the trivial case when the subintervals constitute simply a partition of $[a, b]$. So, if

$$
h_{1}+\cdots+h_{n}=b-a,
$$

taking as coefficients $c_{1}=\cdots=c_{n}=1$ we obtain a formula (of type (1), with $a_{k}=h_{k}$, 
$k=1, \ldots, n)$ which is exact for every integrable function $f$ and, in particular, for all polynomials $p$ of degree $2 n-1$. Moreover, these are the only coefficients that produce a formula of $\mathrm{ADP}=2 n-1$. This follows from the fact that for any fixed set of nonoverlapping subintervals $I_{k}:=\left[x_{k}, x_{k}+h_{k}\right], k=1, \ldots, n$, the coefficients $\left\{c_{k}\right\}$ are uniquely determined by the condition that (1) integrates exactly all polynomials from $\pi_{n-1}$. Indeed, by Proposition 1 , there exist polynomials $p_{k}$ of degree $n-1$ satisfying the conditions $\int_{I_{i}} p_{k}(x) d x=\delta_{i k}$. Then $c_{k}=\int_{a}^{b} p_{k}(x) d x$ and thus the coefficients $\left\{c_{k}\right\}$ are determined uniquely.

In the sequel we assume that the total length of the subintervals is less than the length of $[a, b]$. To simplify the presentation we introduce some notations.

For a given finite interval $[a, b]$, we introduce the set $H$ of admissible lengths $\mathbf{h}=$ $\left(h_{1}, \ldots, h_{n}\right)$,

$$
H:=\left\{\mathbf{h} \in \mathbb{R}^{n}: h_{k} \geq 0, k=1, \ldots, n, \sum_{k=1}^{n} h_{k}<b-a\right\}
$$

and the associated set

$$
D=D(\mathbf{h}):=\left\{\mathbf{x} \in \mathbb{R}^{n}: a<x_{1} \leq x_{1}+h_{1}<\cdots<x_{n} \leq x_{n}+h_{n}<b\right\}
$$

of admissible nodes.

Theorem 3 (General Existence Theorem). Let $\nu_{1}, \ldots, \nu_{n}$ be arbitrary positive even integers and $\sum_{k=1}^{n} \nu_{k}=N$. For any weight function $\mu(t)$ on $[a, b]$ and any set of lengths $\mathbf{h} \in H$, there exists a generalized Gaussian interval quadrature formula of the form

$$
\begin{gathered}
\int_{a}^{b} \mu(t) u(t) d t \approx \sum_{k=1}^{n} \sum_{\lambda=0}^{\nu_{k}-2} a_{k, \lambda} \frac{1}{d_{k}-c_{k}} \int_{c_{k}}^{d_{k}} \mu(t) u(t) v_{\lambda}(t) d t, \\
a \leq c_{1} \leq d_{1} \leq c_{2} \leq d_{2} \leq \cdots \leq c_{n} \leq d_{n} \leq b .
\end{gathered}
$$

with respect to any given Tchebycheff system $u_{1}, \ldots, u_{N}$ of continuous functions. Moreover, its coefficients $a_{i, j}^{*}$ satisfy

$$
a_{k, \nu_{k}-2}^{*}\left\{\begin{array}{l}
>0, \nu_{k}=2 \\
\neq 0, \nu_{k}>2
\end{array}\right.
$$

for $k=1,2, \ldots, n$.

The proof is based on the Borsuk Antipodal Theorem. It can be seen in [3] (Theorem 1 ). Let us give separately the most important particular case, when $\nu_{1}=\cdots=\nu_{n}=2$.

COROLlary 2. Let $u_{1}, \ldots, u_{2 n}$ be any Tchebycheff system of continuous functions on $[a, b]$ and $\mu(t), \nu(t)$ be weight functions on $[a, b]$. Then, for every given system of lengths

$$
h_{1} \geq 0, \ldots, h_{n} \geq 0
$$

there exists a quadrature formula of the form

$$
\int_{a}^{b} \mu(t) u(t) d t \approx \sum_{k=1}^{n} c_{k} \frac{1}{h_{k}^{\circ}} \int_{x_{k}}^{x_{k}+h_{k}} \nu(t) u(t) d t
$$

which is exact for all $u \in \operatorname{span}\left\{u_{1}, \ldots, u_{2 n}\right\}$.

Here $h_{k}^{\circ}:=\int_{x_{k}}^{x_{k}+h_{k}} \nu(t) d t$. 
4.2. Uniqueness. The uniqueness of the Gaussian formula (1) was established first in the particular case when the lengths of all subintervals are equal. The proof in [3] covers the case of weighted integration of arbitrary Tchebycheff space $\mathcal{U}_{2 n}:=\operatorname{span}\left\{u_{1}, \ldots, u_{2 n}\right\}$. We give it below.

THEOREM 4. Let $\mu(t)$ be an arbitrary weight function on $[a, b]$. Then for each natural $n$ and $0<h \leq(b-a) / n$, there exists a unique set of non-overlapping subintervals $\Delta_{k}$, $k=1, \ldots, n$, of the same length $h$ such that the interpolatory quadrature formula

$$
\int_{a}^{b} \mu(t) f(t) d t \approx \sum_{k=1}^{n} A_{k} \int_{\Delta_{k}} \mu(t) f(t) d t
$$

is exact for each $u \in \mathcal{U}_{2 n}$.

Proof. The existence follows from Theorem 3. The main ingredient of the proof of the uniqueness is Proposition 3, concerning interval interpolation in case we allow certain kind of overlapping. Having Proposition 3, the rest of the proof is just an elegant conclusion. We shall sketch it here.

Assume that there are two different quadrature formulae with node subintervals $\left\{I_{k}\right\}$, $\left\{J_{k}\right\}$ and coefficients $\left\{A_{k}\right\},\left\{B_{k}\right\}$, respectively, that are exact for any $u \in \mathcal{U}_{2 n}$. By Theorem $3, A_{k}>0$ and $B_{k}>0$ for all $k$. Since the quadratures are different, then there is at least one subinterval from the first group, say $I_{m}$, which is distinct from every subinterval from the second group $\left\{J_{k}\right\}$. By Proposition 3, there exists a generalized polynomial $w_{m} \in \mathcal{U}_{2 n}$ such that

$$
\int_{I_{m}} \mu(t) w_{m}(t) d t=1, \quad \int_{\Delta} \mu(t) w_{m}(t) d t=0
$$

for any other subinterval $\Delta \neq I_{m}$. Then, by the first quadrature (based on $\left\{I_{j}\right\}$ ),

$$
\int_{a}^{b} \mu(t) w_{m}(t) d t=A_{m}>0
$$

while by the other one (based on $\left\{J_{k}\right\}$ ) the same integral equals 0 . The contradiction shows the uniqueness.

In the pointwise setting the Gauss formula for the algebraic system is closely related to orthogonal polynomials. A similar relation takes place also in the averaged value setting in case the lengths of the subintervals are equal. To explain this relation more precisely, let us assume that $[a, b]=[0,1]$ and $h=1 / m$ with some $m \geq n+1$. Then (see Theorem 4 in [3]) the quadrature formula

$$
\int_{0}^{1} f(x) d x \approx \sum_{k=1}^{n} a_{k} \int_{x_{k}}^{x_{k}+h} f(t) d t
$$

is exact for every $f \in \pi_{2 n-1}$ if and only if the polynomial $\omega(x):=\prod_{k=1}^{n}\left(x-x_{k}\right)$ is orthogonal to any polynomial of degree $\leq n-1$ with respect to the discrete inner product

$$
(f, g):=\sum_{j=0}^{m-1} f(j / m) g(j / m) .
$$

The following uniqueness theorem was established recently in [5]. 
For a given weight function $\mu(t)$ on $[a, b]$, points $\left\{x_{k}\right\}$, and lengths $\mathbf{h} \in H$, let us set

$$
I_{k}[g]:=\int_{x_{k}}^{x_{k}+h_{k}} \mu(t) g(t) d t, \quad I_{k}^{\circ}:=I_{k}[1] .
$$

In case $h_{k}=0$ the quantity $I_{k}[f] / I_{k}^{\circ}$ is defined by continuity, that is,

$$
\left.\frac{1}{I_{k}^{\circ}} I_{k}[f]\right|_{h_{k}=0}:=\lim _{h_{k} \rightarrow 0} \frac{1}{I_{k}^{\circ}} I_{k}[f]=f\left(x_{k}\right) .
$$

We shall consider the Gauss problem with respect to an Extended Tchebycheff system. Let us first recall that $u_{1}, \ldots, u_{2 n}$ is an Extended Tchebycheff system of order $m$ in $[a, b]$ if every non-zero generalized polynomial $a_{1} u_{1}(x)+\cdots+a_{2 n} u_{2 n}(x)$ has at most $2 n-1$ zeros counting multiplicities up to order $m$.

TheOREM 5. Let $u_{1}, \ldots, u_{2 n}$ be any Extended Tchebycheff system of order 2 of continuously differentiable functions on $[a, b]$ and let $\mu$ be an integrable function on $[a, b]$ which is continuous and positive on $(a, b)$. Then, for every given set of numbers $\mathbf{h} \in H$ there exists a unique set of nodes $\mathbf{x} \in D(\mathbf{h})$ such that

$$
\int_{a}^{b} \mu(t) f(t) d t=\sum_{k=1}^{n} a_{k} \frac{1}{I_{k}^{\circ}} \int_{x_{k}}^{x_{k}+h_{k}} \mu(t) f(t) d t
$$

for every $f$ from the space $\mathcal{U}_{2 n}:=\operatorname{span}\left\{u_{1}, \ldots, u_{2 n}\right\}$.

Next we comment on the proof of this uniqueness theorem.

The problem can be reduced (in various ways) to a system of non-linear equations

$$
F_{j}\left(h_{1}, \ldots, h_{n} ; x_{1}, \ldots, x_{n}\right)=0, \quad j=1, \ldots, n,
$$

in unknowns $x_{1}, \ldots, x_{n}$ over $D(\mathbf{h})$, i.e., over the set

$$
a<x_{1} \leq x_{1}+h_{1}<x_{2} \leq x_{2}+h_{2}<\cdots<x_{n} \leq x_{n}+h_{n}<b .
$$

The techniques of proving uniqueness of the solution of a system of non-linear equations are analogues of the techniques used in the univariate case. For example, the usual tool to show that the equation $f(t)=0$ has a unique solution in $[a, b]$ is to check the conditions:

$$
f(a) f(b)<0, \quad f^{\prime}(t)>0 \quad \text { on } \quad(a, b) .
$$

The essential part is the constant sign of the derivative. The multivariate analogue of this is the condition

$$
\operatorname{det}\left\{\frac{\partial F_{j}(\mathbf{x})}{\partial x_{i}}\right\}_{i=1, j=1}^{n}{ }^{n} \neq 0 \quad \forall \mathbf{x} \in D(\mathbf{h}) .
$$

This is a very strong requirement and difficult to check. There is not much chance to prove uniqueness of the solution of interesting systems using the above mentioned simple argument. However, there is another approach which can be applied to a wider class of problems. Its univariate analogue consists of the following: If $f^{\prime}(\xi)>0$ at every point $\xi$ from $(a, b)$ such that $f(\xi)=0$, then $\xi$ is unique. Indeed, if $f$ is increasing at every point where its graph crosses the real axis, then it may cross the axis only once (see Fig. 12). Thus, instead of requiring monotonicity of the function on the whole interval, it is enough to have monotonicity of the same kind only at the zeros. In the multivariate case this 


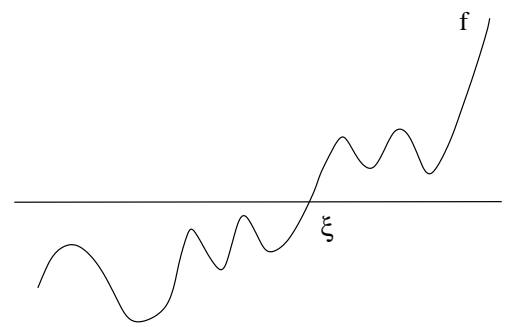

Fig. 12. Non-linear equation

idea is realized through the topological degree of continuous mappings. Let us describe it. Some definitions will be needed.

Let $D$ be a bounded open subset of $\mathbb{R}^{n}$ with closure $\bar{D}$ and boundary $\partial D$. Let the map $\Phi: \bar{D} \rightarrow \mathbb{R}^{n}$ be continuous. For $\mathbf{c} \in \mathbb{R}^{n}$ and $\mathbf{c} \notin \Phi(\partial D)$ we shall denote by $\operatorname{deg}(\Phi, D, \mathbf{c})$ the topological degree of $\Phi$ with respect to $D$ and c (see [24], [29]).

Next we recall some properties of the degree which will be exploited in our proof (see [24], pp. 148 - 162).

(i) Kronecker Theorem. Let $D$ be a bounded open subset of $\mathbb{R}^{n}$ and $\Phi$ a continuous map from $\bar{D}$ into $\mathbb{R}^{n}$. If $\mathbf{c} \notin \Phi(\partial D)$ and if $\operatorname{deg}(\Phi, D, \mathbf{c}) \neq 0$, then the equation $\Phi(\mathbf{x})=\mathbf{c}$ has a solution in $D$.

(ii) Homotopy Invariance Theorem. Let $\Phi(\mathbf{x}, \alpha)$ be a continuous map defined on $\bar{D} \times[0,1]$ with $\Phi(\mathbf{x}, \alpha) \neq \mathbf{c}$ for any $\mathbf{x} \in \partial D, 0 \leq \alpha \leq 1$. Then $\operatorname{deg}(\Phi(\cdot, \alpha), D, \mathbf{c})$ is a constant independent of $\alpha$.

(iii) Suppose that $\Phi \in C^{1}(D), \mathbf{c} \notin \Phi(\partial D)$ and $\operatorname{det}\left(\Phi^{\prime}(\mathbf{x})\right) \neq 0$ for any $\mathbf{x} \in D$ such that $\Phi(\mathbf{x})=\mathbf{c}$. Then there exist only a finite number of points $\mathbf{x}^{(i)}$ for which $\Phi\left(\mathbf{x}^{(i)}\right)=\mathbf{c}$ and

$$
\operatorname{deg}(\Phi, D, \mathbf{c})=\sum_{i} \operatorname{sign} \operatorname{det}\left(\Phi^{\prime}\left(\mathbf{x}^{(i)}\right)\right) .
$$

The method of topological degree can be used to prove existence and uniqueness of the solution of a given non-linear system. To do this one proceeds in the following way. Let $\Phi(\mathbf{x})=\mathbf{0}$ be the system of equations with respect to $\mathbf{x}$ in $D$. Introduce an appropriate family of systems $\Phi(\mathbf{x} ; \alpha)=\mathbf{0}$ parameterized by $\alpha \in[0,1]$ (and depending continuously on $\alpha$ ) so that the system $\Phi(\mathbf{x} ; 0)=\mathbf{0}$ has a unique solution and

$$
\Phi(\mathbf{x} ; 1) \equiv \Phi(\mathbf{x}) .
$$

Assume that $\Phi(\mathbf{x} ; \alpha)$ is a continuously differentiable function of $\mathbf{x}$ in $D$ for each $\alpha \in[0,1]$. Assume that

$$
\begin{aligned}
& \mathbf{0} \notin \Phi(\partial D(\alpha) ; \alpha) \quad \forall \alpha \in[0,1], \\
& \operatorname{det} \Phi^{\prime}(\mathbf{x} ; \alpha)>0 \quad \text { for any } \mathbf{x} \in D \text { such that } \Phi(\mathbf{x} ; \alpha)=\mathbf{0} .
\end{aligned}
$$

Then, according to property (iii) of the degree

$$
\operatorname{deg}(\Phi(\cdot ; 0), D, \mathbf{0})=1 .
$$


Further, by the Homotopy Invariance Theorem

$$
\operatorname{deg}(\Phi(\cdot ; \alpha), D, \mathbf{0})=1 \quad \text { for every } \alpha \in[0,1],
$$

and then, by the Kronecker Theorem, the system $\Phi(\mathbf{x} ; \alpha)=\mathbf{0}$ has a solution for every $\alpha \in[0,1]$. Again by property (iii) this solution is unique. Therefore, the original system

$$
\Phi(\mathbf{x} ; 1)=\Phi(\mathbf{x})=\mathbf{0}
$$

has a unique solution.

The particular case of Theorem 4, dealing with a constant weight $\mu(t)$ and algebraic polynomials, was proved in [4] using the method of topological degree. Actually, the method was used repeatedly, step by step, in order to reach the system of equations defined by preassigned lengths $\mathbf{h}$, starting from the system of equations that corresponds to the classical Gauss formula (i.e., that one defined by lengths $h_{1}=\cdots=h_{n}=0$ ). Let us sketch the idea of proof. As we already mentioned the problem is reduced to the study of a non-linear system of equations

$$
F_{j}(\mathbf{x} ; \mathbf{h}):=F_{j}\left(x_{1}, \ldots, x_{n} ; h_{1}, \ldots, h_{n}\right)=0, \quad j=1, \ldots, n,
$$

in unknowns $x_{1}, \ldots, x_{n}$ over $D(\mathbf{h})$. The lengths $\mathbf{h}$ are fixed. First we introduce a family of maps

$$
\Phi(\mathbf{x} ; \alpha):=\left(F_{1}(\mathbf{h}(\alpha) ; \mathbf{x}), \ldots, F_{n}(\mathbf{h}(\alpha) ; \mathbf{x}), \quad \alpha \in[0,1],\right.
$$

parameterized by $\alpha$, and defined respectively on $D(\mathbf{h}(\alpha))$ where

$$
\mathbf{h}(\alpha):=\left(\alpha h_{1}, \ldots, \alpha h_{n}\right) .
$$

For $\alpha=0$ we get a system of equations that defines uniquely the nodes of the usual Gauss quadrature formula, and consequently one can easily verify that

$$
\operatorname{deg}(\Phi, D(\mathbf{h}(0), \mathbf{0})=1 .
$$

Next we prove that

$$
\operatorname{det} \Phi^{\prime}(\mathbf{x} ; \alpha)>0, \quad \alpha \in[0,1],
$$

at any solution $\mathbf{x}(\alpha)$ of the system $\Phi(\mathbf{x} ; \alpha)=\mathbf{0}$ in $D(\mathbf{h}(\alpha))$. Clearly,

$$
D\left(\mathbf{h}\left(\alpha_{2}\right)\right) \subset D\left(\mathbf{h}\left(\alpha_{1}\right)\right) \text { for } \alpha_{1}<\alpha_{2} .
$$

Thus, it could happen that the unique solution $\mathbf{x}(0)$ corresponding to the system $\Phi(\mathbf{x}, 0)=$ $\mathbf{0}$ does not belong to $D(\mathbf{h}(1))=D(\mathbf{h})$. Then we cannot use the map $\Phi(\mathbf{x} ; 0)$ as initial one in the method of topological degree since the system $\Phi(\mathbf{x} ; 0)=\mathbf{0}$ has no solution in $D(\mathbf{h})$. To overcome this obstacle we prove first that if $\mathbf{h} \in H_{\varepsilon}$ for some $\varepsilon>0$, where

$$
H_{\varepsilon}:=\left\{\mathbf{h} \in H: \sum_{k=1}^{n} h_{k} \leq b-a-\varepsilon\right\},
$$

then there exists an absolute number $\varepsilon_{0}>0$ (depending only on $\varepsilon$ ) such that any solution of the system

$$
F_{j}(\mathbf{x} ; \mathbf{h})=0, \quad j=1, \ldots, n,
$$

satisfies the restrictions

$$
x_{1}-a>\varepsilon_{0}, \quad x_{j+1}-x_{j}-h_{j}>\varepsilon_{0}, j=1, \ldots, n-1, \quad b-x_{n}-h_{n}>\varepsilon_{0} .
$$


Next we notice that if $\mathbf{x}$ is a solution of the system $\Phi(\mathbf{x} ; \alpha)=\mathbf{0}$ for some $\alpha \in[0,1)$, then it belongs to $D(\mathbf{h}(\alpha+\delta)$ ) with sufficiently small $\delta>0$ (which can be computed on the basis of $\varepsilon_{0}$ ). Thus we can apply the method of topological degree starting from $\alpha=0$ and proving existence and uniqueness of the solution for $\alpha=\delta$. Then using this result as initial one we prove the existence and uniqueness of the solution for $\alpha=2 \delta$ and so on, until we reach $\alpha=1$.

This repeatedly used application of the method of topological degree is actually the main contribution of [4] to the study of non-linear systems. It was adopted later in [19] for the proof of the existence and uniqueness of the Gaussian interval quadrature formulae with Jacobi weight function. Numerical algorithms for finding the location of the optimal subintervals were presented in [19].

A crucial moment in the application of the method of topological degree is proving that $\operatorname{det} \Phi^{\prime}(\mathbf{x} ; \alpha) \neq 0$ at the solutions of the corresponding system. This was done in [4] in the case the weight $\mu(t)$ is constant. Technical difficulties occur in the computation of $\operatorname{det} \Phi^{\prime}$ when $\mu(t)$ is an arbitrary weight. Recently, a new system was derived in [5] that describes the node intervals of the Gaussian interval quadrature in the general case, namely, with any weight function and with respect to any fixed Tchebycheff system. For convenience, we shall use the same notations as in the above for this new system and its parametrization $\Phi(\mathbf{x} ; \alpha)$. The specific form of the new equations makes it possible to show that

$$
\operatorname{det} \Phi^{\prime}(\mathbf{x}(\alpha) ; \alpha) \neq 0
$$

for every solution $\mathbf{x}(\alpha)$ of the system $\Phi(\mathbf{x} ; \alpha)=\mathbf{0}$. Thus the proof of the existence and uniqueness of the solution can be completed as in the previous case by a repeated application of the method of topological degree starting from the case $\alpha=0$ that was covered by Krein's theorem. It was noted in [5] however that there is another, simpler and direct way based on the Implicit Function Theorem (IFT). We shall describe it below as a separate proposition.

TheOREM 6. Let $D(\alpha), \alpha \in[0,1]$, be nested bounded open subsets of $\mathbb{R}^{n}$, i.e., such that

$$
D(1) \subset D\left(\alpha_{2}\right) \subset D\left(\alpha_{1}\right) \subset D(0) \text { for } 0 \leq \alpha_{1} \leq \alpha_{2} \leq 1,
$$

and let $D(\alpha)$ depends continuously on $\alpha \in[0,1]$ (i.e., the Hausdorff distance between $D(\alpha)$ and $D(\beta)$ tends to zero as $\alpha \rightarrow \beta)$. Let $\Phi(\mathbf{x}, \alpha)$ be a continuous map defined on $\bar{D}(\alpha) \times[0,1]$ with $\Phi(\mathbf{x}, \alpha) \neq \mathbf{0}$ for any $\mathbf{x} \in \partial D(\alpha), 0 \leq \alpha \leq 1$. Suppose that, for every $\alpha \in[0,1], \Phi \in C^{1}(D(\alpha)), \mathbf{0} \notin \Phi(\partial D(\alpha))$ and $\operatorname{det}\left(\Phi^{\prime}(\mathbf{x})\right) \neq 0$ for any $\mathbf{x} \in D(\alpha)$ such that $\Phi(\mathbf{x} ; \alpha)=\mathbf{0}$. Assume that the system of equations $\Phi(\mathbf{x} ; 0)=\mathbf{0}$ has a unique solution $\mathbf{x}(0)$ in $D(0)$. Then the system $\Phi(\mathbf{x} ; 1)=\mathbf{0}$ has a unique solution in $D(1)$.

Proof. The existence follows from the IFT since the solution $\mathbf{x}(0)$ can be extended to a solution $\mathbf{x}(\alpha)$ of the system $\Phi(\mathbf{x} ; \alpha)=\mathbf{0}$ for any $\alpha, 0 \leq \alpha \leq 1$. Note that $\mathbf{x}(\alpha)$ will belong to $D(\alpha)$ for every $\alpha$. Indeed if, say $\mathbf{x}(\beta)$ is outside $D(\beta)$ for some $\beta \in(0,1]$, then, since $\mathbf{x}(0) \in D(0)$ by assumptions and since $D(\alpha)$ changes continuously with $\alpha$, we should have $\mathbf{x}(\alpha) \in \partial D(\alpha)$ for some $\alpha \in(0, \beta)$. But this contradicts the assumptions.

The uniqueness also follows from the IFT. Indeed, assume that the system $\Phi(\mathbf{x} ; 1)=\mathbf{0}$ has two distinct solutions $\mathbf{x}(1)$ and $\mathbf{y}(1)$. Any of these solutions can be extended uniquely 
to $\mathbf{x}(\alpha), \mathbf{y}(\alpha)$ for every $\alpha$ starting from 1 and going back to 0 . On one hand, $\mathbf{x}(1) \neq \mathbf{y}(1)$, and on the other hand, according to the assumption, $\mathbf{x}(0)=\mathbf{y}(0)$. Let $\beta$ be the largest number in $[0,1)$ for which $\mathbf{x}(\beta)=\mathbf{y}(\beta)$. Then there must be two distinct extensions of $\mathbf{x}(\alpha)$ in a neighborhood of $\beta$ which contradicts the IFT. The proof is complete.

The proof of Theorem 4 now follows immediately as an application of the method presented in Theorem 5 introducing the parameterized family of systems corresponding to $\mathbf{h}(\alpha):=\left(\alpha h_{1}, \ldots, \alpha h_{n}\right)$, for $\alpha \in[0,1]$, and taking into account that Krein's theorem guarantees the existence and uniqueness of the solution in the initial case $\alpha=0$.

The following particular case of Theorem 5 deserves to be mentioned.

COROLlary 3. Let $\mu$ be any integrable function on $[a, b]$ which is continuous and positive on $(a, b)$. Then, for every given set of non-negative numbers $\mathbf{h}$ satisfying the condition

$$
h_{1}+\cdots+h_{n} \leq b-a,
$$

there exists a unique set of nodes $\mathbf{x} \in D(\mathbf{h})$ and coefficients $\left\{a_{k}\right\}$ such that

$$
\int_{a}^{b} \mu(t) f(t) d t=\sum_{k=1}^{n} a_{k} \frac{1}{I_{k}^{\circ}} \int_{x_{k}}^{x_{k}+h_{k}} \mu(t) f(t) d t
$$

for every algebraic polynomial of degree less than or equal to $2 n-1$.

Acknowledgments. I am grateful to Petar Petrov and to an anonymous referee who read the manuscript with care and made very useful suggestions. Thanks also go to Irina Georgieva who helped me with the figures.

\section{References}

[1] B. Bojanov, A note on the Hobby-Rice and Gauss-Krein theorems, East J. Approx. 4 (1998), 371-377.

[2] B. Bojanov, H. Hakopian and A. Sahakian, Spline Functions and Multivariate Interpolations, Kluwer Academic Publishers, Dordrecht, 1993.

[3] B. Bojanov and P. Petrov, Gaussian interval quadrature formula, Numer. Math. 87 (2001), 625-643.

[4] B. Bojanov and P. Petrov, Uniqueness of the Gaussian interval quadrature formula, Numer. Math. 95 (2003), 53-62.

[5] B. Bojanov and P. Petrov, Gaussian interval quadrature formula for a Tchebycheff system, SIAM J. Numer. Anal. 43 (2005), 787-795.

[6] B. Bojanov and G. Petrova, On minimal cubature formulae for product weight functions, J. Comp. Appl. Math. 85 (1997), 113-121.

[7] B. Bojanov and G. Petrova, Numerical integration over a disc. A new Gaussian quadrature formula, Numer. Math. 80 (1998), 39-59.

[8] B. Bojanov and G. Petrova, Uniqueness of the Gaussian cubature for a ball, J. Approx. Theory 104 (2000), 21-44.

[9] B. Bojanov and I. Georgieva, Interpolation by bivariate polynomials based on Radon projections, Studia Mathematica 162 (2004), 141-160.

[10] B. Bojanov and Y. Xu, On polynomial interpolation of two variables, J. Approx. Theory 120 (2003), 267-282. 
[11] B. Bojanov and Y. Xu, Reconstruction of a polynomial from its Radon projections, SIAM J. Math. Anal. 37 (2005), 238-250.

[12] A. S. Cavaretta, C. A. Micchelli and A. Sharma, Multivariate interpolation and the Radon transform, Part I, Math. Z. 174 (1980), 263-279; Part II, in: Quantitive Approximation, R. DeVore and K. Scherer (eds.), Acad. Press, New York, 1980, 49-62.

[13] Ch. F. Dunkl and Yuan Xu, Orthogonal Polynomials of Several variables, Cambridge University Press, Cambridge, 2001.

[14] C. Gauss, Methodus Nova Integralium Valores per Approximationem Inveniendi, Commentationes Societatis Regiae Scientiarum Gottingensis Recentiores 3 (1814), Werke III, $163-196$.

[15] H. Hakopian, Multivariate divided differences and multivariate interpolation of Lagrange and Hermite type, J. Approx. Theory 34 (1982), 286-305.

[16] M. Krein, The ideas of P. L. Tchebycheff and A. A. Markov in the theory of limiting values of integrals and their further developments, Uspekhi Fiz. Nauk (1951), 3-120 (Russian); Amer. Math. Soc. Transl. Ser. 2, 12 (1951), 1-122.

[17] A. L. Kuz'mina, Interval quadrature formulae with multiple node intervals, Izv. Vuzov 7(218) (1980), 39-44 (in Russian).

[18] R. Marr, On the reconstruction of a function on a circular domain from a sampling of its line integrals, J. Math. Anal. Appl. 45 (1974), 357-374.

[19] G. V. Milovanović and A. C. Cvetković, Uniqueness and computation of Gaussian interval quadrature formula for Jacobi weight function, Numer. Math. 99 (2004), 141-162.

[20] C. R. Morrow and T. N. L. Patterson, Construction of algebraic cubature rules using polynomial ideal theory, SIAM J. Numer. Anal. 15 (1978), 953-976.

[21] V. P. Motornyi, On the best quadrature formulae in the class of functions with bounded r-th derivative, East J. Approx. 4 (1998), 459-478.

[22] I. Mysovskih, Interpolatory Cubature Formulas, Nauka, Moscow, 1981 (in Russian).

[23] M. Omladic, S. Pahor and A. Suhadolc, On a new type quadrature formulas, Numer. Math. 25 (1976), 421-426.

[24] J. M. Ortega and W. C. Rheinboldt, Iterative Solutions of Nonlinear Equations in Several Variables, Academic Press, New York, 1970.

[25] K. Oskolkov, On representation of algebraic polynomials by superpositions of plane waves, Serdica Math. J. 28 (2002), 379-390.

[26] Fr. Pittnauer and M. Reimer, Interpolation mit Intervallfunktionalen, Math. Z. 146 (1976), $7-15$.

[27] Fr. Pittnauer and M. Reimer, Intervallfunktionale vom Gauss-Legendre-Type, Math. Nachr. 87 (1979), 239-248.

[28] R. N. Sharipov, Best interval quadrature formulae for Lipschitz classes, in: Constructive Function Theory and Functional Analysis, Kazan University, Kazan, 1983, Issue 4, 124132 (in Russian).

[29] T. J. Schwartz, Nonlinear Functional Analysis, Gordon and Breach, New York, 1969.

[30] B. A. Vostretsov and M. A. Kreines, On the approximation of continuous functions by supperpositions of plane waves, Dokl. AN SSSR 140 (1961), 1237-1240 (in Russian).

[31] Y. Xu, Lagrange interpolation on Chebyshev points of two variables, J. Approx. Theory 87 (1996), 220-238. 